\title{
PERCOLATION ON GRIGORCHUK GROUPS
}

\author{
Roman Muchnik and Igor Pak \\ Department of Mathematics, \\ Yale University, \\ New Haven, CT 06520
}

December 21, 1999

\begin{abstract}
Let $p_{c}(G)$ be the critical probability of the site percolation on the Cayley graph of group $G$. In [2] of Benjamini and Schramm conjectured that $p_{c}<1$, given the group is infinite and not a finite extension of $\mathbb{Z}$. The conjecture was proved earlier for groups of polynomial and exponential growth and remains open for groups of intermediate growth.

In this note we prove the conjecture for a special class of Grigorchuk groups, which is a special class of groups of intermediate growth. The proof is based on an algebraic construction. No previous knowledge of percolation is assumed.
\end{abstract}

\section{Introduction}

While percolation on $\mathbb{Z}^{d}$ has been studied for decades, percolation on general Cayley graphs became popular only in the past several years. This direction of research was outlined in an important paper [2] of Benjamini and Schramm. The authors conjectured that the critical probability $p_{c}$ for the site percolation satisfies

$$
p_{c}<1
$$

given the group is infinite and not a finite extension of $\mathbb{Z}$.

Since appearance of [2], a number of interesting results has been obtained. The above conjecture has been established for groups of polynomial or exponential growth, as well as for finitely presented groups (see 
$[1,3,23])$. In this note we prove the conjecture for a class of so called Grigorchuk groups, which are defined as groups of Lebesgue-measure-preserving transformations on the unit interval. Recently other examples of groups of intermediate growth were found, notably Gupta-Sidki groups and Grigorchuk $p$-groups (see $[5,9,16,17])$. These are the only known examples of groups of intermediate growth ${ }^{1}$.

Let $G$ be an infinite group generated by a finite set $S, S=S^{-1}$, and let $\Gamma=\Gamma(G, S)$ be the corresponding Cayley graph. Consider Bernoulli site percolation in which all vertices are independently open with probability $p$ (closed with probability $1-p)$. By $\theta(p)$ denote the probability $P(i d \leftrightarrow \infty)$, i.e. the probability that the open cluster containing $i d$ is infinite. Define the critical probability

$$
p_{c}(G)=\sup \{p: \theta(p)=0\}
$$

It is known that $p_{c}(G)>1 /|S|, p_{c}\left(\mathbb{Z}^{2}\right)=1 / 2$, and that $p_{c}\left(G_{1}\right) \leq p_{c}\left(G_{2}\right)$ if $G_{2}$ is a subgroup or a quotient group of $G_{1}$. We refer to [12, 13] for a thorough treatment of classical percolation, and to $[2,3]$ for the many interesting questions and results for percolation on Cayley graphs.

Conjecture (Benjamini and Schramm)

If $\Gamma$ is the Cayley graph of an infinite (finitely generated) group $G$, which is not a finite extension of $\mathbb{Z}$, then $p_{c}<1$.

The conjecture is one of the central unsolved questions of the percolation theory on Cayley graphs. While it was established for groups of exponential and polynomial growth (see $[3,23]$ ), the conjecture remains open for groups of intermediate growth. The conjecture has been also confirmed for finitely presented groups (see [1]).

Let us remark here that the analogous conjecture for bond percolation is equivalent to Conjecture (see e.g. [12, 13]). Recently Häggström also showed equivalence of the conjecture with having a phase transition for Ising and Widom-Rowlinson models (see [19]).

Let $\omega=\left(\omega_{1}, \omega_{2}, \ldots\right)$ be an infinite sequence of elements in the set $\{0,1,2\}$. Grigorchuk group $G_{\omega}$ is a infinite profinite 2-group whose construction depends on $\omega$ (see $[8,11]$ ). Groups $G_{\omega}$ are generated by 4 involutions, while the structure and even the growth is different for different $\omega$. We postpone definition of $G_{\omega}$ till the next section.

Theorem 1. The conjecture holds for Grigorchuk groups $G_{\omega}$ for all $\omega$.

\footnotetext{
${ }^{1}$ Of course, other groups of intermediate growth can be constructed from these groups. Study of percolation then can be reduced to the latter.
} 
The proof relies on growth considerations and a fractal structure of Grigorchuk groups. Essentially, we find $\mathbb{Z}_{+}^{2}$ in the corresponding Cayley graphs. More precisely, we prove that $G_{\omega}$ contains a subgroup of finite index which is isomorphic to $A_{\omega} \times B_{\omega}$, where $A_{\omega}, B_{\omega}$ are infinite finitely generated groups. From here we show that conjecture holds for $A_{\omega} \times B_{\omega}$, and therefore for $G_{\omega}$.

Let us conclude by saying that in a sequel paper [9] Grigorchuk introduced a wider class of groups which correspond to sequences of $0,1, \ldots, p$, where $p$ is a prime. Analogously, Gupta and Sidki in $[16,17]$ constructed different series of $p$-groups, some of them later proved to have intermediate growth (see [5]). While we do not consider these groups, it is not hard to see that our analysis can be directly translated to these cases.

\section{Growth of groups and percolation}

Before we define the $G_{\omega}$ and prove the theorem, let us comment on the growth of groups and it's relevance to percolation problems.

Let $G$ be an infinite group generated by a finite set $S, S=S^{-1}$, and let $\Gamma=\Gamma(G, S)$ be the corresponding Cayley graph. Let $B(n)$ be the set of elements $g \in G$ at a distance $\leq n$ from $i d$ in graph $\Gamma$. The growth function of $G$ with respect to the set of generators $S$ is defined as $\gamma(n)=|B(n)|$.

We say that a function $f: \mathbb{N} \rightarrow \mathbb{R}$ is dominated by a function $g: \mathbb{N} \rightarrow \mathbb{R}$, denoted by $f \preccurlyeq g$, if there is a constant $C>0$ such that $f(n) \leq g(C \cdot n)$ for all $n \in \mathbb{N}$. Two functions $f, g: \mathbb{N} \rightarrow \mathbb{R}$ are called equivalent, denoted by $f \sim g$, if $f \preccurlyeq g$ and $g \preccurlyeq f$. It is known that for any two finite sets of generators $S_{1}, S_{2}$ of a group $G$, the corresponding two growth functions are equivalent (see e.g. [21, 28]). Note also that if $|S|=k$, then $\gamma(n) \leq k^{n}$.

Growth of group $G$ is called exponential if $\gamma(n) \sim e^{n}$. Otherwise the growth is called subexponential. For example, all nonamenable groups have exponential growth (but not vice versa.) Growth of group $G$ is called polynomial if $\gamma(n) \sim n^{c}$ for some $c>0$. Otherwise the growth is called superpolynomial.

We call a group $G$ almost nilpotent (solvable, etc.) if it contains a nilpotent (solvable, etc.) subgroup of finite index. The celebrated result of Gromov implies that groups of polynomial growth must be virtually nilpotent (see [15]). We add that by the Tits alternative all linear groups must be either almost solvable or contain a free group $F_{2}$. Finally, the Milnor-Wolf theorem states that all solvable groups have either polynomial or exponential growth (see [21, 28]). 
If the growth of $G$ is subexponential and superpolynomial, it is called intermediate. In a pioneer paper [7] Grigorchuk disproved Milnor's conjecture by exhibiting a group of intermediate growth. In [8] he defined a class of groups and proved that they have growth

$$
\exp \left(n^{\alpha_{1}}\right) \preccurlyeq \gamma(n) \preccurlyeq \exp \left(n^{\alpha_{2}}\right)
$$

for some $1 / 2 \leq \alpha_{1}, \alpha_{2}<1$.

A sequence $\omega=\left(\omega_{1}, \omega_{2}, \ldots\right)$ is called constant if $\omega_{1}=\omega_{2}=\ldots$ Otherwise $\omega$ is called non-constant. It is not hard to see that if $\omega$ is constant, then $G_{\omega} \cong\left\{a, b \mid a^{2}=b^{2}=i d\right\}$ (see section 3). Thus $G_{\omega}$ is $\mathbb{Z}_{2} * \mathbb{Z}_{2}$ and the conjecture holds. If $\omega$ is non-constant we will show that the natural Cayley graph in this case contains $\mathbb{Z}_{+}^{2}$ as a subgraph, which proves the theorem. Note that under mild conditions $G_{\omega}$ is a torsion group, so it does not contain $\mathbb{Z}^{2}$ as a subgroup.

A sequence $\omega=\left(\omega_{1}, \omega_{2}, \ldots\right)$ is called stabilizing if $\omega_{N}=\omega_{N+1}=\ldots$ for some $N$. We remark here that if $\omega$ is stabilizing sequence, then Grigorchuk group $G_{\omega}$ has an intermediate growth (see [8, 20, 27]).

Let us return to percolation on Cayley graphs. Let us first note that if the critical percolation $p_{c}$ is strictly less than 1 for some generating set, the same is true for all generating sets. While we could not find this precise result in the literature, we believe it to be known and refer to [25] for similar coupling arguments.

Now recall that when the growth of $G$ is polynomial, $G$ is virtually nilpotent. We have the following result.

Theorem 2. (Benjamini) If $\Gamma$ is a Cayley graph of an infinite group $G$ of polynomial growth which is not a finite extension of $\mathbb{Z}$, then $p_{c}(G)<1$.

The case when the growth of $G$ is exponential was solved in [23]. First, observe that the natural Cayley graph of a free group $F_{k}, k \geq 2$ is isomorphic to regular $2 k$-ary tree, where the percolation is well understood (see e.g. [24]). While the general exponential groups do not have to contain $F_{k}$, their Cayley graphs have been shown to contain a tree with positive branching number (see [22, 23]), which proves that conjecture in this case.

Let us briefly outline the Lyons' argument. Fix a lexicographic ordering on generators in $S$ and connect each element $g \in G$ with $i d$ by a path which corresponds to the reduced words of minimum length. This gives a spanning subtree $T$ in the Cayley graph. Now, the exponential growth function of $G$ gives the positive Minkowski dimension of $T$, which by the famous result of Furstenberg implies positive Hausdorff dimension of $T$ (see $[6,22])$. The 
latter implies that $T$ has critical probability $<1$ and proves the following theorem (see $[22,23])$.

Theorem 3. (Lyons) If $G$ has exponential growth, then $p_{c}(G)<1$.

As a corollary we obtain the $p_{c}(G)<1$ for almost solvable and linear group, which are not almost $\mathbb{Z}$, as well as for Burnside group $B(n, p)$, where $p>661$ (see $[21,28]$ ). Let us also mention here a different result of Grigorchuk that if $\gamma(n) \preccurlyeq e^{\sqrt{n}}$, and $G$ is residually finite $p$-group, then $G$ has polynomial growth (see [10]). This confirms Conjecture 1 for such groups as well.

A few words about group presentation. First, it is known that when $\omega$ is non-stabilizing, the Grigorchuk group $G_{\omega}$ is not finitely presented (see $[8,26])$. Thus Theorem 1 does not follow from results in [1] for finitely presented groups. Moreover, it was conjectured by Adian that all finitely presented groups must have either polynomial or exponential growth (see [11]). Further, it was conjectured by Grigorchuk that all finitely presented groups must either contain $F_{2}$ or be virtually nilpotent. Even if (weaker) Adian conjecture holds, this would prove that results of [1] are inapplicable to groups of intermediate growth.

\section{Grigorchuk Group}

In this section we will describe a construction of Grigorchuk's 2-group. For a complete description and further results see [8].

Let $\Delta$ be an interval. Denote by $I$ an identity transformation on $\Delta$ and by $T$ a transposition of two halves of $\Delta$.

Let $\Omega$ be a set of infinite sequences $\omega=\left(\omega_{1}, \omega_{2}, \ldots\right)$ of elements of the set $\{0,1,2\}$. For each $\omega \in \Omega$ define a $3 \times \infty$ matrix $\bar{\omega}$ by replacing $\omega_{i}$ with columns $\bar{\omega}_{i}$ where

$$
\overline{0}=\left(\begin{array}{c}
T \\
T \\
I
\end{array}\right), \overline{1}=\left(\begin{array}{c}
T \\
I \\
T
\end{array}\right), \overline{2}=\left(\begin{array}{c}
I \\
T \\
T
\end{array}\right)
$$

By $U^{\omega}=\left(u_{1}^{\omega}, u_{2}^{\omega}, \ldots\right), V^{\omega}=\left(v_{1}^{\omega}, v_{2}^{\omega}, \ldots\right), W^{\omega}=\left(w_{1}^{\omega}, w_{2}^{\omega}, \ldots\right)$ denote the rows of $\bar{\omega}$. Think of them as of infinite words in the alphabet $\{T, I\}$.

Define transformations $a_{\omega}, b_{\omega}, c_{\omega}, d_{\omega}$ of an interval $\Delta=[0,1] \backslash \mathbb{Q}$ as follows: 

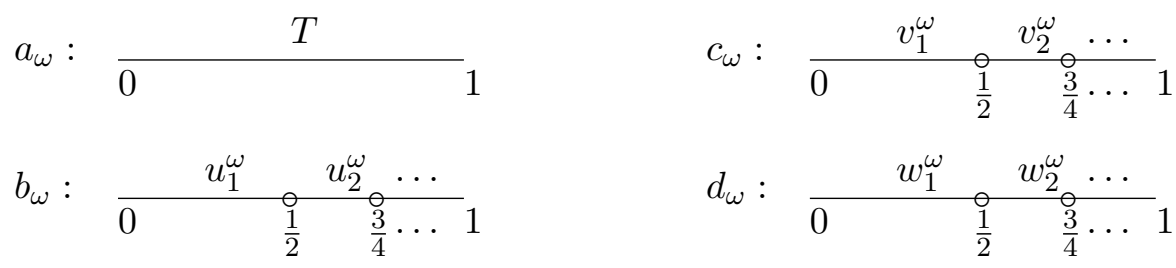

Observe that $a_{\omega}$ is independent of $\omega$, and will be further denoted by a. Let $G_{\omega}$ be a group of transformations of the interval $\Delta$ generated by $a, b_{\omega}, c_{\omega}, d_{\omega}$. This family of groups was introduced and analyzed by Grigorchuk in [8] (see also [20] for further references). We refer to $G_{\omega}$ as Grigorchuk groups.

Observe that the generators of $G_{\omega}$ satisfy the following relations:

$$
\begin{gathered}
a^{2}=b_{\omega}^{2}=c_{\omega}^{2}=d_{\omega}^{2}=1, \\
c_{\omega} b_{\omega}=b_{\omega} c_{\omega}=d_{\omega}, \\
d_{\omega} b_{\omega}=b_{\omega} d_{\omega}=c_{\omega}, \\
c_{\omega} d_{\omega}=d_{\omega} c_{\omega}=b_{\omega},
\end{gathered}
$$

We call these simple relations. Under mild conditions, the groups $G_{\omega}$ are known to be not finitely presented (see $[8,20]$ ).

Denote by $\Gamma_{\omega}$ a Cayley graph of the group $G_{\omega}$ with respect to the generators $a, b_{\omega}, c_{\omega}, d_{\omega}$. For every element $g \in G_{\omega}$ by $\partial(g)$ denote the smallest distance between $g$ and $i d$ in $\Gamma_{\omega}$. The paths in $\Gamma_{\omega}$ correspond to words in the alphabet $\left\{a, b_{\omega}, c_{\omega}, d_{\omega}\right\}^{*}$. The shortest paths (there could be many of them between two given elements) correspond to the reduced words in the alphabet. Recall that the balls in the Cayley graph $\Gamma_{\omega}$ are defined as $B_{\omega}(n)=\left\{g \in G_{\omega} \mid \partial(g) \leq n\right\}$.

Let $\sigma: \Omega \rightarrow \Omega$ be a right shift operator acting on the infinite sequences as follows

$$
\sigma:\left(\omega_{1}, \omega_{2}, \omega_{3}, \ldots\right) \rightarrow\left(\omega_{2}, \omega_{3}, \ldots\right)
$$

Denote by $\Delta_{0}$ and $\Delta_{1}$ the half intervals $\Delta \cap[0,1 / 2]$ and $\Delta \cap[1 / 2,1]$. Define $H_{\omega} \subset G_{\omega}$ to be a stabilizer of $\Delta_{0}$. Clearly, $g: \Delta_{0} \rightarrow \Delta_{0}$ and $g: \Delta_{1} \rightarrow \Delta_{1}$ for all $g \in H_{\omega}$.

Define $\phi_{0}^{\omega}: H_{\omega} \rightarrow G_{\sigma \omega}$ by restricting $h \in H_{\omega}$ to $\Delta_{0}$. Formally, while $H_{\omega}$ acts on $\Delta_{0}$ rather than $\Delta$ we can rescale the interval to obtain transformations in $G_{\sigma \omega}$. Similarly define $\phi_{1}^{\omega}: H_{\omega} \rightarrow G_{\sigma \omega}$ by restricting to $\Delta_{1}$ and then rescaling to the unit interval. 
It is easy to see that $H_{\omega}$ is a normal subgroup of index 2 , which is generated by 6 elements $b_{\omega}, c_{\omega}, d_{\omega}, a b_{\omega} a, a c_{\omega} a, a d_{\omega} a$.

We will omit superscript $\omega$ in $\phi_{0,1}^{\omega}$ when it is clear on which $H_{\omega}$ the map $\phi_{i}^{\omega}$ acts. The following table summarizes the images of homomorphisms of $\phi_{0}, \phi_{1}$ on the generators of subgroup $H_{\omega}$.

TABLE

\begin{tabular}{|c|r|c|c|c|c|r|}
\hline & $b_{\omega}$ & $c_{\omega}$ & $d_{\omega}$ & $a b_{\omega} a$ & $a c_{\omega} a$ & $a d_{\omega} a$ \\
\hline$\phi_{0}$ & $u_{1}^{\omega}$ & $v_{1}^{\omega}$ & $w_{1}^{\omega}$ & $b_{\sigma \omega}$ & $c_{\sigma \omega}$ & $b_{\sigma \omega}$ \\
\hline$\phi_{1}$ & $b_{\sigma \omega}$ & $c_{\sigma \omega}$ & $b_{\sigma \omega}$ & $u_{1}^{\omega}$ & $v_{1}^{\omega}$ & $w_{1}^{\omega}$ \\
\hline
\end{tabular}

\section{Lifted subgroups}

Let $F_{1}, F_{2} \subset H_{\omega}$ be subgroups which act trivially on $\Delta_{0}$ and $\Delta_{1}$ respectively:

$$
\begin{gathered}
F_{1}=\left\{h \in H_{\omega} \mid h \equiv I \text { on } \Delta_{0}\right\} \\
F_{2}=\left\{h \in H_{\omega} \mid h \equiv I \text { on } \Delta_{1}\right\} .
\end{gathered}
$$

Define a subgroup $D_{\omega}=F_{1} \cdot F_{2}$. Observe that $F_{1}$ commutes with $F_{2}$, $F_{1} \cap F_{2}=\{i d\}$, and $D_{\omega} \simeq F_{1} \times F_{2}$. Also, let $F \simeq F_{1}=a F_{2} a \simeq F_{2}$.

Lemma $1 .^{2} D_{\omega}$ is a normal subgroup of $G_{\omega}$.

Proof. Observe that

$$
\begin{gathered}
a\left(F_{1} \cdot F_{2}\right) a=a F_{1} a \cdot a F_{2} a=F_{2} \cdot F_{1}=F_{1} \cdot F_{2}, \\
b_{\omega}\left(F_{1} \cdot F_{2}\right) b_{\omega}=b_{\omega} F_{1} b_{\omega} \cdot b_{\omega} F_{2} b_{\omega}=F_{1} \cdot F_{2}, \\
c_{\omega}\left(F_{1} \cdot F_{2}\right) b_{\omega}=c_{\omega} F_{1} c_{\omega} \cdot c_{\omega} F_{2} c_{\omega}=F_{1} \cdot F_{2}, \\
d_{\omega}\left(F_{1} \cdot F_{2}\right) b_{\omega}=d_{\omega} F_{1} d_{\omega} \cdot d_{\omega} F_{2} d_{\omega}=F_{1} \cdot F_{2}
\end{gathered}
$$

Since $a, b_{\omega}, c_{\omega}, d_{\omega}$ are the generators in $G_{\omega}$, we obtain $g D_{\omega} g^{-1}=D_{\omega}$ for all $g \in G$. This proves the result.

Lemma 2. If $\omega$ is non-constant, $D_{\omega}$ has a finite index in $G_{\omega}$.

\footnotetext{
${ }^{2}$ It was pointed out to us by the referee that in [18] a related result on subgroups was proved. The referee suggests that this might lead to a generalization of our main result. We challenge the reader to obtain such a generalization.
} 
Proof. Since $\omega$ is non-constant, there exist an integer $k$ such that $\omega_{k} \neq$ $\omega_{1}$. Without loss of generality assume that $\omega_{1}=0, \omega_{k}=1$, and $k$ is the first 1 in $\omega$. To simplify the notation denote $s=k-1$.

Since $D_{\omega}$ is a normal subgroup in $G_{\omega}$, consider a natural map to the quotient group

$$
\pi: G_{\omega} \rightarrow G_{\omega} / D_{\omega}
$$

Observe that $\pi\left(d_{\omega}\right)=i d, \pi\left(c_{\omega}\right)=\pi\left(b_{\omega} d_{\omega}\right)=\pi\left(b_{\omega}\right)$. Therefore $\pi(G)=$ $\pi\left(\left\langle a, c_{\omega}\right\rangle\right)$. We will prove below that the subgroup $\left\langle a, c_{\omega}\right\rangle$ has a finite order. This immediately implies that the quotient group has a finite order. Thus $D_{\omega}$ has a finite index which proves the Lemma.

We claim that the element $a c_{\omega} \in G_{\omega}$ has a finite order. This immediately implies that the order of subgroup $\left\langle a, c_{\omega}\right\rangle \subset G$ is finite.

The proof by induction on $k$ any for any sequence $\tau$, such that $\tau_{k}=1$. For $k=1$, from the TABLE we have

$$
\phi_{0}\left(a c_{\tau} a c_{\tau}\right)=c_{\sigma \tau} \text { and } \phi_{1}\left(a c_{\tau} a c_{\tau}\right)=c_{\sigma \tau} .
$$

By definition the element $c_{\sigma \tau}$ has order 2. Therefore $a c_{\tau}$ has order 4 .

Assume $a c_{\tau}$ has finite order for any $k \leq n$, where $\tau_{k}=1$. For $k=n+1$, we have

$$
\phi_{0}\left(a c_{\tau} a c_{\tau}\right)=c_{\sigma \tau} a \text { and } \phi_{1}\left(a c_{\tau} a c_{\tau}\right)=a c_{\sigma \tau} .
$$

By induction hypothesis for $\sigma \tau$ we conclude that each element on the right hand side has a finite order. Therefore $a c_{\tau}$ has a finite order. This finishes step of induction and proves the claim.

Corollary 1. If $\omega$ is non-constant, then $F_{1} \simeq F_{2}$ is infinite.

Proof. By Lemma $2 D_{\omega} \simeq F_{1} \times F_{2}$ has a finite index in an infinite group $G_{\omega}$. This implies the result.

\section{$5 \quad$ Proof of Theorem 1.}

By results in section 3, we need to consider only the case when $\omega$ is nonconstant. We will prove that $p_{c}\left(G_{\omega}\right)<1$. The proof of this claim follows from the results in the previous section.

Indeed, consider a percolation on $D=D_{\omega}$. We claim that $p_{c}(D)<1$. Recall from the previous section that $D \simeq F_{1} \times F_{2}$ and both $F_{1}$ and $F_{2}$ are infinite. We need the following simple result. 
Lemma 3. Let $A$ and $B$ be two infinite finitely generated groups. Then $p_{c}(A \times B)<1$.

The lemma immediately proves Theorem 1 . Indeed, $p_{c}(G) \leq p_{c}(D)<1$ which finishes the proof.

Proof of Lemma 3. Let $S_{1}=\left\{a_{1}, \ldots, a_{n}\right\}$ and $S_{2}=\left\{b_{1}, \ldots, b_{m}\right\}$ be any generating sets of $A$ and $B$ respectively. Assume that $S_{1}=S_{1}^{-1}, S_{2}=S_{2}^{-1}$. Denote by $\Gamma_{1}, \Gamma_{2}$ the corresponding Cayley graphs.

Observe that the set $\left\{\left(a_{1}, 1\right), \ldots,\left(a_{n}, 1\right),\left(1, b_{1}\right), \ldots,\left(1, b_{m}\right)\right\}$ generates $A \times B$. The corresponding Cayley graph $\Gamma$ is isomorphic to a direct product $\Gamma_{1} \times \Gamma_{2}$.

Since $A$ is infinite group we can choose an infinite self-avoiding path $\left(x_{1}, x_{2}, \ldots\right) \subset \Gamma_{1}$ (i.e. path with distinct vertices.) Analogously, we can choose an infinite self-avoiding path $\left(y_{1}, y_{2}, \ldots\right) \subset \Gamma_{2}$.

Now consider a spanning subgraph $\Xi$ in $\Gamma$ with vertices $\left(x_{i}, y_{j}\right) \in A \times B$. By construction $\Xi \supset \mathbb{Z}_{+}^{2}$. Therefore $p_{c}(A \times B) \leq p_{c}(\Xi) \leq p_{c}\left(\mathbb{Z}_{+}^{2}\right)$. Recall that $p_{c}\left(\mathbb{Z}_{+}^{2}\right)<1$ (see [12]). This finishes the proof.

\section{Odds and ends}

Using a weak version of the axiom of choice one can conclude that the Cayley graph of $G_{\omega}$ contains $\mathbb{Z}^{2}$. Simply choose bi-directed paths in both Cayley graphs $\Gamma_{1}, \Gamma_{2}$ and let the length go to infinity.

In a different direction, a beautiful proof of Burton and Keane in [4] implies that for all amenable groups the infinite percolation cluster is almost surely (a.s.) unique. Now, using a more complicated construction of lifted subgroups (going to the next level) one can show that the Cayley graph of $G_{\omega}$ contains $\mathbb{Z}_{+}^{4}$. While similar, the proof is somewhat more involved and will be omitted. As a corollary we conclude that the (a.s. unique) percolation cluster is a.s. transient when $p>p_{c}$. This follows from the transience of the percolation cluster on $\mathbb{Z}^{3}$ (see $[13,14]$ ). Note also that for Gupta-Sidki groups a similar construction of lifted subgroups gives immediately $\mathbb{Z}_{+}^{3}$. We leave the details to the reader.

As an ultimate challenge to the reader we ask about the behavior of the critical percolation on Grigorchuk groups. We refer to $[2,3,13]$ for the references and details. 


\section{ACKNOWLEDGEMENTS.}

We are grateful to Itai Benjamini, Hillel Furstenberg, Russell Lyons, Gregory Margulis, Yuval Peres, Alexander Retakh, Oded Schramm, Tatiana Smirnova-Nagnibeda and Efim Zelmanov for useful remarks. Comment by the referee were also helpful.

The second named author would like to thank the National Science Foundation Postdoctoral Research Fellowship for financial support.

\section{References}

[1] E. Babson, I. Benjamini, Cut sets and normed cohomology with applications to percolation, Proc. Amer. Math. Soc., 127 (1999), no. 2, $589-597$

[2] I. Benjamini, O. Schramm, Percolation beyond $\mathbb{Z}^{d}$, many questions and a few answers, Electron. Comm. Probab., 1 (1996), 71-82

[3] I. Benjamini, O. Schramm, Recent progress on percolation beyond $\mathbb{Z}^{d}$, a survey (1999)

[4] R. M. Burton. M. Keane, Density and uniqueness in percolation, Comm. Math. Phys., 121 (1989), 501-505

[5] J. Fabrykowski, N. Gupta, On groups with sub-exponential growth functions. II. J. Indian Math. Soc. (N.S.) 56 (1991), no. 1-4, 217-228

[6] H. Furstenberg, Disjointness in ergodic theory, minimal sets, and a problem in Diophantine approximation, Math. Systems Theory 1 (1967), 1-49

[7] R. I. Grigorchuk, On the Milnor problem of group growth, Soviet Math. Dokl. 28 (1983), no. 1, 23-26

[8] R. I. Grigorchuk, Degrees of growth of finitely generated groups and the theory of invariant means, Math. USSR-Izv. 25 (1985), no. 2, 259-300

[9] R. I. Grigorchuk, Degrees of growth of $p$-groups and torsion-free groups, Math. USSR-Sb. 54 (1986), no. 1, 185-205

[10] R. I. Grigorchuk, On the Hilbert-Poincaré series of graded algebras that are associated with groups, Math. USSR-Sb. 66 (1990), no. 1, 211-229 
[11] R. I. Grigorchuk, P. F. Kurchanov, Some questions of group theory related to geometry, in Algebra. VII. Combinatorial group theory. Applications to geometry, Encyclopaedia of Mathematical Sciences, vol. 58, Springer, Berlin, 1993

[12] G. R. Grimmett, Percolation, Springer, New York, 1989

[13] G. R. Grimmett, Percolation and disordered systems, in Lectures on Probability Theory and Statistics, Saint-Flour (1996), Lecture Notes 1665, Springer, Berlin

[14] G. R. Grimmett, H. Kesten, Y. Zhang, Random walk on the infinite cluster of the percolation model, Probab. Theory Related Fields, 96 (1993), 33-44

[15] M. Gromov, Groups of polynomial growth and expanding maps, Inst. Hautes Études Sci. Publ. Math. No. 53 (1981), 53-73

[16] N. Gupta, S. Sidki, On the Burnside problem for periodic groups, Math. Z. 182 (1983), no. 3, 385-388

[17] N. Gupta, S. Sidki, Some infinite p-groups, Algebra i Logika 22 (1983), no. $5,584-589$

[18] N. Gupta, S. Sidki, Extension of groups by tree automorphisms, in Contributions to group theory, 232-246, Contemp. Math., 33, Amer. Math. Soc., Providence, R.I., 1984.

[19] O. Häggström, Markov random fields and percolation on general graphs, to appear in Advances in Appl. Probab., (1998).

[20] P. de la Harpe, Topics on Geometric Group Theory, book in preparation (1998)

[21] G. R. Krause, T. H. Lenagan, Growth of algebras and Gelfand-Kirillov dimension, Research Notes in Mathematics, 116, Pitman, Boston, 1985

[22] R. Lyons, Random walks and percolation on trees, Ann. Probab., 18 (1990), 931-958

[23] R. Lyons, Random walks and the growth of groups, C. R. Acad. Sci. Paris Sir. I Math., 320 (1995), 1361-1366

[24] R. Lyons, Y. Peres, Probability on trees and networks, book in preparation (1998) 
[25] R. Lyons, O. Schramm, Indistinguishability of percolation clusters, to appear in Ann. Probab. (1998)

[26] I. G. Lysenok, A set of defining relations for the Grigorchuk group, Math. Notes 38 (1985), 784-792

[27] R. Muchnik, I. Pak, Growth of Grigorchuk groups, to appear in Intern. J. Algebra and Comp., (2000)

[28] V. A. Ufnarovskij, Combinatorial and asymptotic methods in algebra, in Algebra, VI, 1-196, Encyclopaedia Math. Sci., 57, Springer, Berlin, 1995 